\title{
On the role of activation and particle-emission data for reaction model validation
}

\author{
M. Avrigeanu ${ }^{1}$, R.A. Forrest ${ }^{2}$, A.J. Koning ${ }^{3}$, F.L. Roman ${ }^{1}$, and V. Avrigeanu ${ }^{1, a}$ \\ 1 EURATOM-MEdC Fusion Association, "Horia Hulubei” National Institute for Physics and Nuclear Engineering, P.O. Box MG-6, \\ 76900 Bucharest, Romania \\ 2 EURATOM-UKAEA Fusion Association, Culham Science Centre, Abingdon OX14 3DB, UK \\ 3 Nuclear Research and Consultancy Group NRG, P.O. Box 25, 1755 ZG Petten, The Netherlands
}

\begin{abstract}
Experimental cross sections are compared with the results of calculations including all activation channels for the stable isotopes of $\mathrm{Mn}$ and $\mathrm{Cu}$, for neutron incident energies below $20 \mathrm{MeV}$ as well as up to $40-50 \mathrm{MeV}$. It results that within the former energy range, the model calculations are most sensitive to the parameters related to nuclei in the early stages of the reaction, while the model assumptions are better proved by analysis of the data in the latter energy range.
\end{abstract}

\section{Introduction}

As part of a general investigation [1-4] of the reaction mechanisms of fast neutrons at low and medium energies, we have analyzed the activation cross sections of the odd-mass isotopes ${ }^{55} \mathrm{Mn}$ and ${ }^{63,65} \mathrm{Cu}$ in the excitation-energy range up to $50 \mathrm{MeV}$.

The main purpose of this work is to present new experimental results and discuss some of the question marks associated with the model calculations which combine preequilibrium emission (PE) with equilibrium decay of the remaining compound nucleus. Although our primary aim was to comply with the needs of a sound, complete and reliable neutron-induced cross section data library to address safety and environmental issues of the fusion program [5,6], the analysis results also enabled a stringent test of models for the above-mentioned nuclear processes. The odd-mass target nuclei within the present work may be particularly useful in connection with the proved influence of the $f_{7 / 2}$ neutron and proton shell closures on the particle PE spectra (e.g., [7]). Actually Koning and Duijvestijn [8] pointed out that omission of the shell effects is probably the most important cause of the remaining discrepancies in their large-scale comparison of the nucleon PE model with angle-integrated nucleon spectra. Moreover, a systematic analysis of Mills et al. [9] in the same mass range highlighted that some discrepancies observed in the yields of nuclides with closed or nearly-closed nucleon shells may not affect the inherent validity of the relevant model, but follow the use of incorrect, average or model parameters for certain nuclei involved in the decay process. This is why, in order to gain insight into this problem, we have analyzed the activation cross sections of ${ }^{55} \mathrm{Mn}$ and ${ }^{63,65} \mathrm{Cu}$ isotopes using the parameter databases obtained previously by global optimization within the computer codes TALYS [8] and EMPIRE-II [10] as well as a local parameter set within the STAPRE-H code $[11,12]$. No fine tuning was done to optimize the description of the nucleon emission for all cases, but for STAPRE-H a consistent set of local parameters has

\footnotetext{
${ }^{a}$ Presenting author, e-mail: vavrig@ifin.nipne.ro
}

been established or validated on the basis of independent experimental information of, e.g., neutron total cross sections, proton reaction cross sections, low-lying level and resonance data, and $\gamma$-ray strength functions based on neutron-capture data. The comparison of various calculations, including their sensitivity to model approaches and parameters, has concerned all activation channels for which there are measured data. The use of model parameters which have been improperly adjusted to take into account properties peculiar to specific nuclei in the decay cascade, considered to be the case for discrepancies observed around the closures of both the $f_{7 / 2}$ proton and neutron shells [9], are thus avoided in the local analysis and may contribute to the model validation.

\section{Nuclear models and calculations}

The two sets of global calculations within the direct-reaction, $\mathrm{PE}$ and statistical Hauser-Feshbach (HF) models, performed by means of the computer codes TALYS [8] and EMPIRE-II [10], have mainly used systematics based on global phenomenological analysis. Thus their results are firstly predictions of the reaction cross sections which should be considered from the point of view of the global parameters involved in the corresponding calculations. However, the performance of the corresponding global estimations is also quite important for large-scale data evaluations based on nuclear model calculations. The main assumptions and parameters involved in this work for both sets of global calculations have been also recently described [4], among other available options, while more detailed descriptions have lately been given $[8,10]$. Therefore we give here only some specific points which have arisen in the meantime as, e.g., the use of the new version (0.72) of the TALYS code and version 2.19 of the code EMPIRE-II. The Hybrid Monte-Carlo simulation approach was used in the latter case for the PE component due to the actual interest for the neutron energies higher than $30 \mathrm{MeV}$ [10]. On the other hand, it has also been possible to take the advantage of using the PE exciton model for cluster emission, i.e., the PCROSS $=1$ option. 


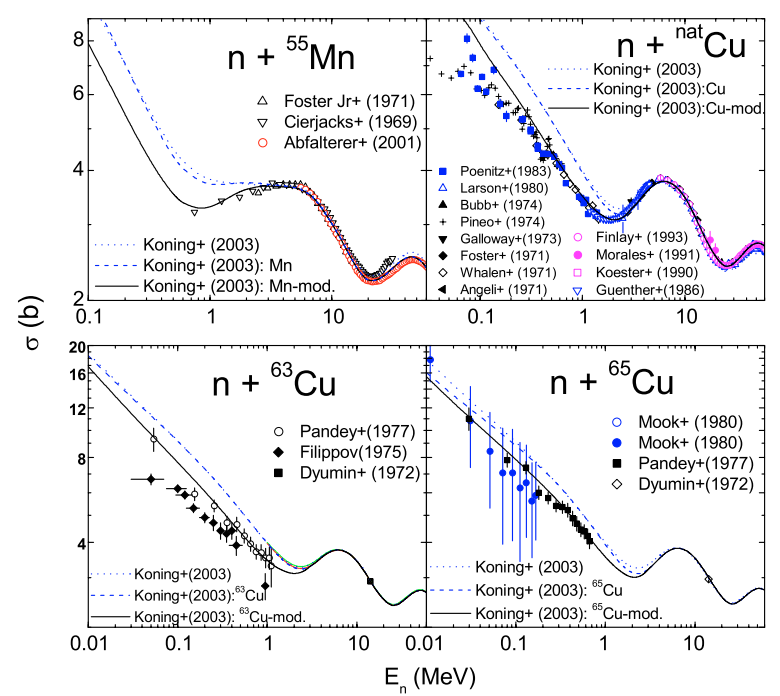

Fig. 1. Comparison of experimental [22] and calculated neutron total cross sections for ${ }^{55} \mathrm{Mn}$ and ${ }^{63,65 \text {, nat }} \mathrm{Cu}$ target nuclei, using the global (dotted curves) and local (dashed curves) OMP parameter sets of Koning and Delaroche [19], and changes of the latter at energies $<3-5 \mathrm{MeV}$ (solid curves).

The particular properties of various target nuclei and reaction channels have been considered using a consistent local parameter set within the code STAPRE-H. A generalized Geometry-Dependent Hybrid (GDH) model $[13,14]$ for PE processes inside this version of the original code STAPRE [11] includes angular-momentum conservation [15] and the $\alpha$ particle and deuteron emission based on a pre-formation probability $\varphi[16]$ with the values in the present work of 0.2 for $\alpha$-particles and 0.4 for deuterons [2]. The same optical model potential (OMP) and nuclear level density parameters have been used in the framework of the GDH and HF models, for calculation of the intra-nuclear transition rates and singleparticle level densities at the Fermi level [14,17,18], respectively, in the former case. The GDH model is characterized by a sum of contributions due to different entrance channel impact parameters for the first projectile-target interaction, with the relevant parameters being averaged over the nuclear densities corresponding to the entrance-channel trajectories instead of the entire nucleus. A composite formula [18] of particle-hole state densities, which trigger the composite nucleus equilibration, is another distinct point of STAPRE-H code.

\section{Local-approach consistent parameter set}

Koning and Delaroche [19] revealed that their neutron OMP, used by default in both TALYS and EMPIRE codes, does not reproduce the minimum around the neutron energy of $1-2 \mathrm{MeV}$ for the total neutron cross sections of the $A \sim 60$ nuclei. Following also their comment on the constant geometry parameters which may be responsible for this aspect, we have applied the SPRT method [20] using the recent RIPL-2 recommendations [21] for the low-energy neutron scattering properties, and the available measured neutron total cross sections (fig. 1). Thus we found that it is suitable

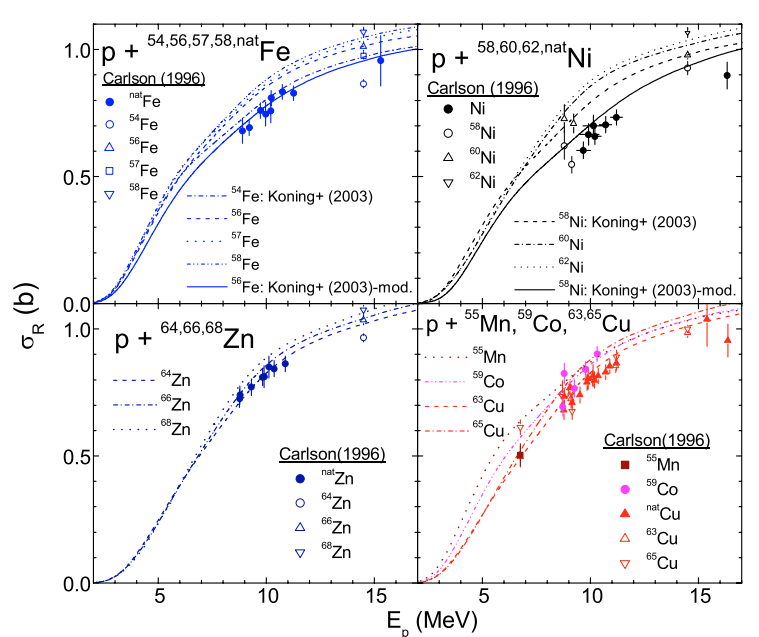

Fig. 2. Comparison of the measured [23] and calculated proton reaction cross sections on all stable isotopes of $\mathrm{Mn}, \mathrm{Fe}, \mathrm{Co}, \mathrm{Ni}, \mathrm{Cu}$ and $\mathrm{Zn}$ elements, using either the local or global OMPs of Koning and Delaroche [19] (dotted, dash-dotted and dashed curves) and a modified parameter set for the target nuclei ${ }^{56} \mathrm{Fe}$ and ${ }^{58} \mathrm{Ni}$ (solid curves).

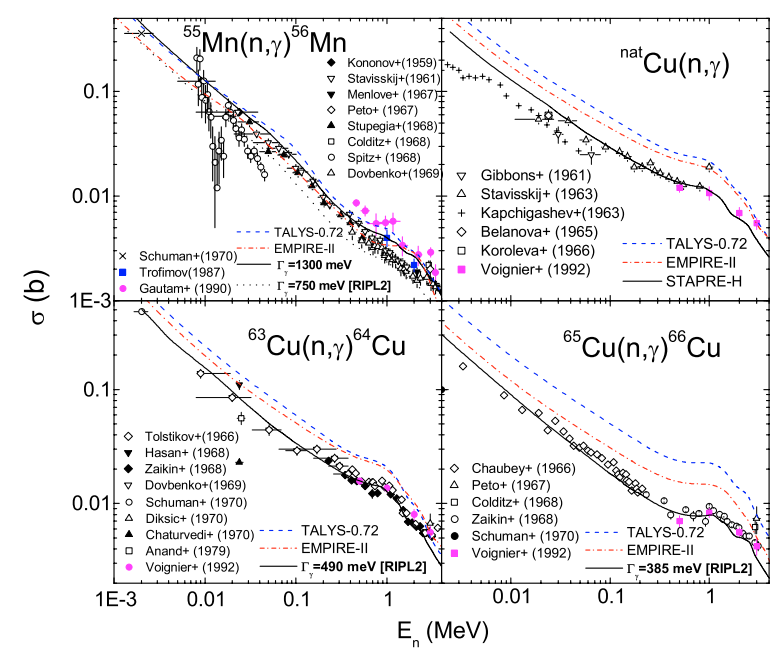

Fig. 3. Comparison of measured [22] neutron-capture cross sections of ${ }^{55} \mathrm{Mn}$ and ${ }^{63,65 \text {,nat }} \mathrm{Cu}$, and calculated values using the codes TALYS-0.72 and EMPIRE-II with default global parameters, and local analysis with EDBW $\gamma$-ray strength functions related to either experimental $\Gamma_{\gamma 0}$ [21] (dotted), or the neutron capture data (solid).

to consider an energy-dependent real potential geometry at energies $<3-5 \mathrm{MeV}$. We made use of these potentials also for calculation of the related DWBA collective inelastic scattering cross sections, with typical ratios to the total reaction cross sections from few to $60 \mathrm{MeV}$ decreasing from $\sim 11$ to $3 \%$.

The OMP of Koning and Delaroche was considered also for the calculation of proton transmission coefficients on the residual isotopes of $\mathrm{Cr}$ and $\mathrm{Ni}$, respectively. Since the particular interest of this work is on activation cross sections, a former trial of this potential concerned the proton reaction cross sections $\sigma_{R}$ using the large compilation of Ref. [23]. The comparison of these data and the results of either the local or otherwise global OMPs [19] is shown in figure 2. A very good 

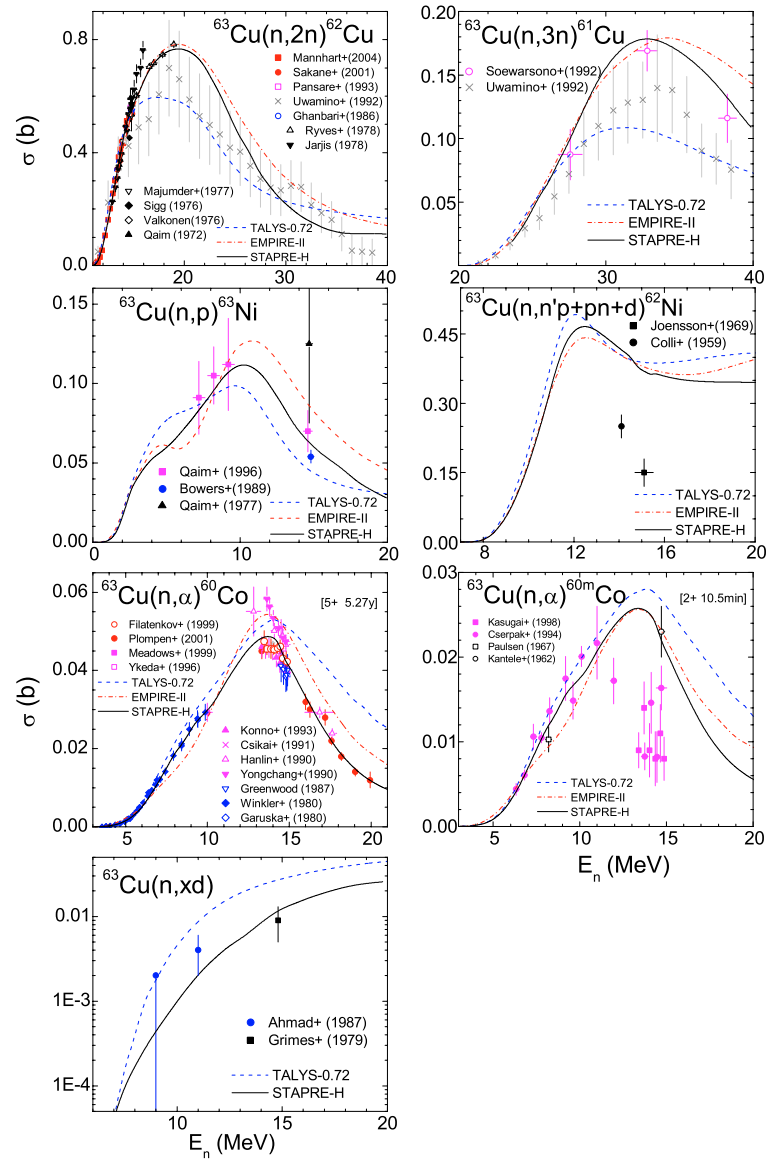

Fig. 4. Same as figure 3, for neutron-activation cross sections of ${ }^{63} \mathrm{Cu}$.

agreement exists apart from the isotopes of $\mathrm{Fe}$ and in particular $\mathrm{Ni}$, with the data overpredicted by about or higher than $10 \%$. By taking again into account an energy dependence of the real potential diffusivity, we have obtained the agreement with the corresponding $\sigma_{R}$ data also shown in figure 2 .

The modified energy-dependent Breit-Wigner (EDBW) model [24,25] was involved for the electric dipole $\gamma$-ray strength functions $f_{E 1}\left(E_{\gamma}\right)$ of main importance for calculation of the $\gamma$-ray transmission coefficients. The systematic correction factors $F_{S R}$ within the EDBW formula were obtained using the experimental [21] average radiative widths $\Gamma_{\gamma 0}^{\exp }$ of the $s$-wave neutron resonances for various nuclei. Moreover, the $f_{E 1}\left(E_{\gamma}\right)$ thus obtained have been checked within the calculations of capture cross sections of $\mathrm{Mn}$ and $\mathrm{Cu}$ isotopes in the neutron energy range from $\mathrm{keV}$ to $3-4 \mathrm{MeV}$ (fig. 3).

\section{Results and discussion}

The experimental cross sections are compared with the results of model calculations for all activation channels in figures 4-6. modified OMPs on the calculated reaction cross sections. The large amount of data existing for the $(n, 2 n)$ and $(n, p)$ reactions on ${ }^{65} \mathrm{Cu}$ has been involved in this respect as it is shown in figure 7 . Thus, firstly one may note that the modified neutron potential of Koning and Delaroche [19] is leading to a decrease of $\sim 5 \%$ for the $(n, 2 n)$ reaction calculated cross sections. At the same time, the modified proton potential of Koning
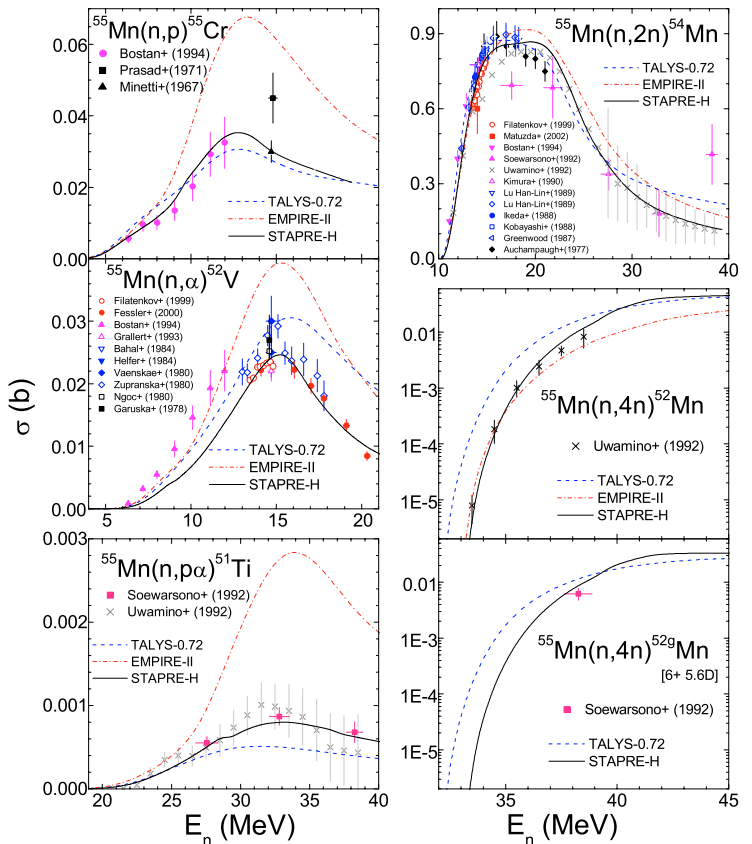

Fig. 5. Same as figure 3, for neutron-activation cross sections of ${ }^{55} \mathrm{Mn}$.
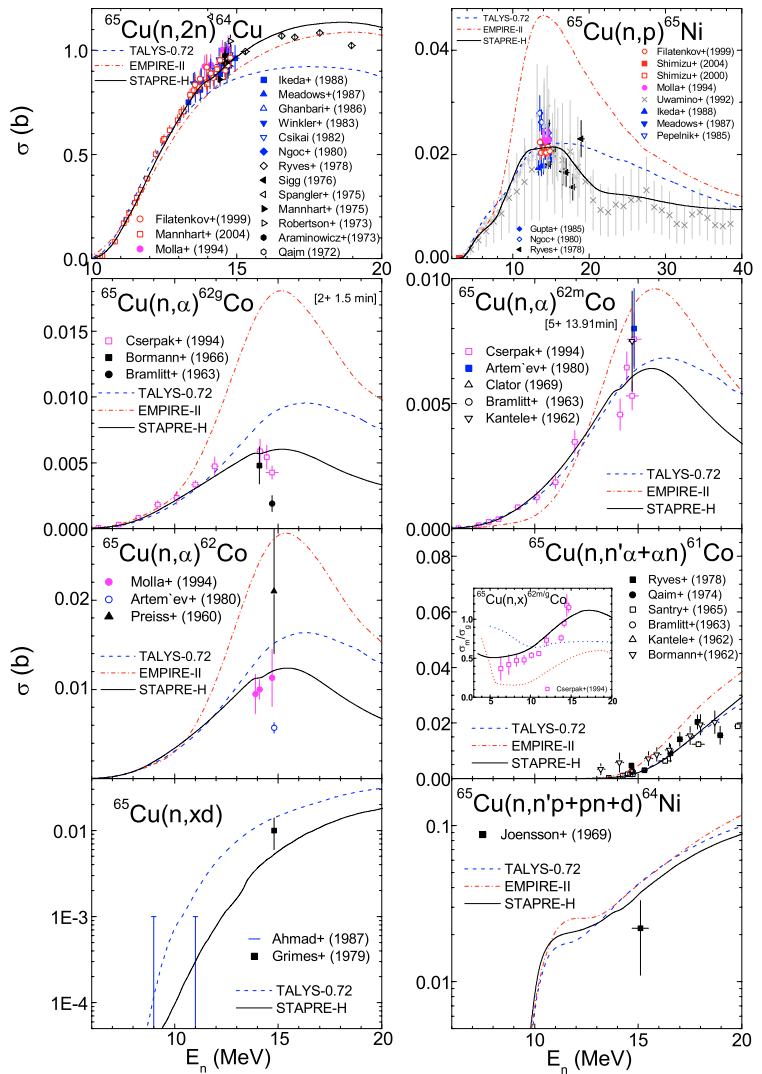

Fig. 6. Same as figure 3, for neutron-activation cross sections of ${ }^{65} \mathrm{Cu}$.

and Delaroche led to a decrease of $\sim 20 \%$ of the calculated $(n, p)$ reaction cross sections. On the other hand, joining together the two changes in the case of the $(n, p)$ reaction, results in a compensation of the latter one and a reduced final change of $\sim 10 \%$ for the calculated cross sections. However, the full effect of each of the two modified OMP could be met, 

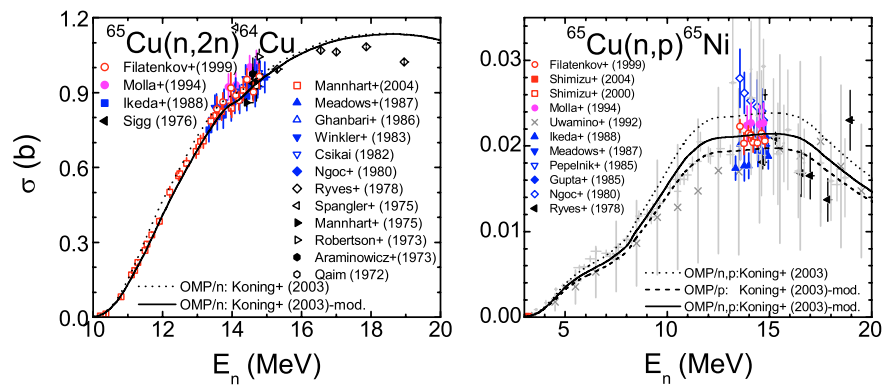

Fig. 7. Comparison of measured [22] and calculated cross sections of the (n, 2n) and (n, p) reactions on ${ }^{65} \mathrm{Cu}$, by using the OMP of Koning and Delaroche [19] (dotted curves), and its local changes within this work for the proton OMP (dashed) as well as neutron OMP (solid).

e.g., within the corresponding calculated particle-emission spectra. Finally one may note that the additional analysis of the nucleon OMP improved the accuracy of the calculated cross sections from $20 \%$, for the smaller cross sections, to around 5\% for the major reaction channels. Actually, just this better precision would be closer to the confined range of 3-4\% accuracy proved by the new measured cross sections around $14 \mathrm{MeV}$. Since also the PE fraction of the total reaction cross section is of only $\sim 15 \%$ at these energies, the two increased accuracies would make possible an effective trial of statistical model parameters acting in that instant or through the PE formalism. Otherwise, the mixture of more effects of similar size, at the same time with less suitable option for one of them, would lead to improper assertion for another one.

In the same respect we may note the following point. By comparison of the present calculations and measured data [22] for the target nuclei ${ }^{63,65} \mathrm{Cu}$, we found that the real well diffuseness of the global OMP [26] for emitted $\alpha$-particles should be decreased to $0.67 \mathrm{fm}$. Since this reduction is rather similar to that found recently to be needed for the target nuclei ${ }^{59} \mathrm{Co}$ and ${ }^{58,60,62} \mathrm{Ni}$ [2], it has been taken into account also for the target nucleus ${ }^{55} \mathrm{Mn}$. However there are a couple of key points related to this matter. First, using just the global OMP [26] for emitted $\alpha$-particles, one would obtain also within the local approach similar results as provided for ${ }^{55} \mathrm{Mn}$ by EMPIRE-II and in very good agreement with the measured data. On the other hand, there is no way to explain the rest of corresponding data above the incident energy of $12 \mathrm{MeV}$, by pure statistical including PE emission. Alternatively one may consider the possible enhancement related to the position of a giant quadrupole resonance at the excitation energies concerned in these nuclei (e.g., ref. [27]).

Following the discussion of the OMP effects on the calculated cross sections, of the order of 5-20\% around the incident energy of $14 \mathrm{MeV}$, one may note the same level of differences at these energies between the global predictions and the measured cross sections. Larger divergence occurs merely at higher energies, where it could be related to the continuously growing importance of PE assumptions and key quantities. However, just the assessment within this energy range of the consistent parameter set involved in the local approach stands for a further focus on differences between the measured and calculated cross sections especially above $20 \mathrm{MeV}$, in order to establish the correctness of the adopted PE formalism.

Work supported in part by the European Communities EFDA under the contract of Association EURATOM/MEdC-Bucharest.

\section{References}

1. P. Reimer, V. Avrigeanu, A.J.M. Plompen, S.M. Qaim, Phys. Rev. C 65, 014604 (2001).

2. V. Semkova et al., Nucl. Phys. A 730, 255 (2004).

3. P. Reimer et al., Phys. Rev. C 71, 044617 (2005).

4. V. Avrigeanu, R. Eichin, R.A. Forrest, H. Freiesleben, M. Herman, A.J. Koning, K. Seidel, Nucl. Phys. A 765, 1 (2006).

5. R.A. Forrest, J. Kopecky, Fus. Eng. Design 82, 73 (2007).

6. R.A. Forrest, J. Kopecky, J.-Ch. Sublet, UKAEA Culham Science Centre Report UKAEA FUS 535, 2007.

7. M. Blann et al., Phys. Rev. C 28, 1475 (1983).

8. A.J. Koning, M.C. Duijvestijn, Nucl. Phys. A 744, 15 (2004).

9. S.J. Mills et al., in Proc. Int. Conf. on Nuclear Data for Science and Technology, Jülich, 1991, edited by S.M. Qaim (SpringerVerlag, Berlin, 1992), p. 708.

10. M. Herman et al., in Proc. Int. Conf. on Nuclear Data for Science and Technology (ND2004), Santa Fe, 2004, edited by R.C. Haight et al., (American Institute of Physics, New York, 2005), p. 1184; EMPIRE-II v.2.19, http://www-nds.iaea.org/empire/.

11. M. Uhl, B. Strohmaier, IRK-Vienna Report IRK-76/01, 1981.

12. M. Avrigeanu, V.Avrigeanu, IPNE Report NP-86-1995, Bucharest, 1995; News NEA Data Bank 17, 22 (1995).

13. M. Blann, H.K. Vonach, Phys. Rev. C 28, 1475 (1983).

14. M. Blann, Nucl. Phys. A 213, 570 (1973).

15. M. Avrigeanu et al., Z. Phys. A 329, 177 (1988); 335, 299 (1990).

16. E. Gadioli, E. Gadioli-Erba, Z. Phys. A 299, 1 (1981).

17. M. Avrigeanu, V. Avrigeanu, J. Phys. G: 20, 613 (1994).

18. M. Avrigeanu, V. Avrigeanu, Comp. Phys. Comm. 112, 191 (1998); A. Harangozo, I. Stetcu, M. Avrigeanu, V. Avrigeanu, Phys. Rev. C 58, 295 (1998).

19. A.J. Koning, J.P. Delaroche, Nucl. Phys. A 713, 231 (2003).

20. J.P. Delaroche, Ch. Lagrange, J. Salvy, IAEA-Vienna Report IAEA-190 (1976), vol. 1, p. 251.

21. IAEA-CRP Reference Input Parameter Library (RIPL-2), http://www-nds.iaea.or.at .

22. EXFOR Nuclear Reaction Data, http://www-nds.iaea.or.at/exfor.

23. R.F. Carlson, At. Data Nucl. Data Tables 63, 93 (1996).

24. D.G. Gardner, F.S. Dietrich, LLNL-Livermore Report UCRL82998, 1979.

25. M. Avrigeanu et al., Rev. Roum. Phys. 32, 837 (1987).

26. V. Avrigeanu, P.E. Hodgson, M. Avrigeanu, Phys. Rev. C 49, 2137 (1994).

27. M. Avrigeanu, W. von Oertzen, V. Avrigeanu, Nucl. Phys. A 764, 246 (2006). 